\title{
Effects of Polyethylenimine Dosages and Molecular Weights on Flocculation, Rheology and Consolidation Behaviors of Kaolin Slurries
}

Lavanya Avadiar $^{\mathrm{a}, \mathrm{c}}$, Yee-Kwong Leong ${ }^{\mathrm{a}, *}$, Andy Fourie ${ }^{\mathrm{b}}$

${ }^{a}$ School of Mechanical and Chemical Engineering

${ }^{\mathrm{b}}$ School of Civil and Resource Engineering

The University of Western Australia,

35 Stirling Highway,

Crawley WA 6009, Australia

bfourie@civil.uwa.edu.au

cavadil01@student.uwa.edu.au

*Corresponding author. Phone number: +618 6488 3602, Fax number: +618 6488 1024,

E-mail address: yeekwong.leong@uwa.edu.au

${ }^{1}$ Abbreviations

\footnotetext{
${ }^{1}$ Polyethylenimine (PEI); Molecular Weights (Mw); Daltons (Da); Dry Weight Percentages (dwb\%); Weight Percentages (wt\%); Cryogenic Scanning Electron Microscopy (cryoSEM); Angstroms ( $\AA$ ); Nephelometric Turbidity Units (NTU); Equivalent Volume Mean Diameters (D $(4,3))$.
} 


\section{Abstract}

There is a lack of literature explaining effects of polyethylenimine (PEI) flocculants on the flocculation and consolidation behaviors of kaolin slurries. In this study, effects of dosages and molecular weights (Mw) of branched PEI polymers on the flocculation, rheology and consolidation aspects of $8 \mathrm{wt} \%$ Unimin kaolin slurries at $\mathrm{pH} 8$ are investigated by conducting yield stress, sedimentation and shear (raking) measurements. Behaviors were supported by zeta potential, particle sizing, turbidity analyses and cryogenic scanning electron microscopy (cryoSEM) imaging, relating to dominant inter-aggregate forces and floc microstructures that governed slurry behaviors. With the addition of low PEI dosages at 0.04 dry weight percentages ( $\mathrm{dwb} \%)$ and $0.004 \mathrm{dwb} \%$, inter-aggregate forces and thus rheological and consolidation behaviors of kaolin slurries were similar to that when no PEI was added. This is attributed to the insufficient amounts and adsorptions of PEI particles onto kaolin particles at low PEI dosages. However, with high PEI dosages of $0.4 \mathrm{dwb} \%$ added, yield stress peaks were significantly higher and solids content obtained were lower compared to slurries without PEI flocculants. This is due to polymer bridging interactions and/or polymer-polymer chain attractions between PEI segments as dominant inter-aggregate forces that resulted in the formation of strong and voluminous flocs, affecting the slurry consolidation and rheological behaviors at high PEI dosages. Unlike PEI dosages, variations in branched PEI Mw caused negligible effects on these behaviors. A correlation is established between PEI dosages, flocculation mechanisms, inter-aggregate forces, floc microstructures and rheological and consolidation behaviors of Unimin kaolin slurries.

Keywords: Kaolinite; Branched polyethylenimine; Polymer bridging interactions; Polymer-polymer chain attractions; Consolidation; Floc microstructures 


\section{Introduction}

The general lack of understanding of the relationship between kaolin platelet-platelet interactions and particle packing density within mineral tailings has caused significant problems to the mining and minerals processing industry [1-3]. Weak and dispersed microstructures formed by these negatively charged particles via van der Waals forces of attraction and hydrogen bonding have resulted in poor dewatering of tailings $[4,5]$. To address this issue, studies have been carried out on the effects of polymeric flocculants on the consolidation behaviors of kaolin slurries [2, 3, 6-11].

Polymer mediated flocculation is understood to improve the dewatering behavior of tailings $[2,9,12]$. These flocculants usually bridge between colloidal particles via positive-negative charge interactions and/or van der Waals forces of attraction to destabilize colloidal particles into aggregation [1]. This initially results in the formation of small flocs that grow with time to be sufficiently dense or large and as a result, undergo sedimentation [1]. Flocculants can be classified according to their molecular weights (Mw), structures (branched or linear), charge densities and configurations, where the latter two factors may depend on the $\mathrm{pH}$ and ionic strength of slurries. Numerous studies concerning kaolin flocculation with high Mw linear polymers that comprise of up to several million Daltons (Da) or $\mathrm{g} / \mathrm{mol}$ have been conducted. In this paper, the effects of dosages and $\mathrm{Mw}$ of branched, positively charged polyethylenimine (PEI) polymers on the consolidation behaviors of kaolin slurries were analyzed.

Literature explaining the effects of branched PEI flocculants on the consolidation of kaolin particles, compared to flocculants such as polyacrylamide and polyethylene oxide, is scarce. Low Mw, branched PEI molecules of high charge densities could adsorb whole on negatively charged faces of kaolin particles, leading to those PEI molecules undergoing electrostatic patch-charge attractions with another kaolin particle at its negatively charged face. High Mw, 
branched polymer molecules could attach themselves onto several kaolin particles via greater extents of bridging interactions [13, 14]. However, Gregory [15], Horn [16] and Alince and Van de Ven [17] suggested that positively charged polymers, such as PEI flocculants, would adopt rather flat configurations on negatively charged particle surfaces due to strong electrostatic attractions where it was expected that such attractions would significantly reduce chances of polymer bridging interactions. These inferences are, however, questionable as there remain probabilities of bridging interactions occurring during collisions between particles and polymers (especially under high particle-polymer concentrations) and during collisions while polymers relax before they reach equilibrium states on particles. Gill and Herrington [18] studied the sedimentation of kaolin slurries with low Mw PEI polymers (2000 g/mol) at $\mathrm{pH} 5$ and identified electrostatic interactions as the primary mechanism causing kaolin particle/floc destabilization. They, however, also made inferences that with high Mw polymer chains, bridging interactions might be the primary mechanism that induced flocculation within these kaolin slurries [18]. Such variations in flocculation mechanisms induced by PEI flocculants of different Mw could affect floc microstructures formed and as a result, kaolin slurry consolidation behaviors.

The effects of PEI flocculants on the consolidation behaviors of kaolin slurries were also studied via their rheological effects on these slurries. Rheological behaviors of nonNewtonian fluids provide an understanding of inter-particle forces that govern microstructures of colloidal particles and that, in turn, explains consolidation behaviors of tailings $[2-4,19,20]$. With most research focusing on either consolidation or rheological aspects of kaolin slurries, there is a lack of literature linking these behavioral aspects of kaolin slurries with PEI flocculants [7, 9, 18, 21, 22].

The effects of shear applied via the introduction of revolving rakes within consolidated sediment beds were also analyzed in this paper. McFarlane et al. [19] reported significant 
improvements in final solids content obtained with post-sedimentation shear forces applied within sediment beds. According to Du et al. [23] and Farrow et al. [24], this is due to the expulsion of water from inter-aggregate and intra-aggregate pore spaces that exist between and within pre-flocculated kaolin aggregates respectively. Hogg [1] suggested that shear forces allowed redistribution and re-arrangement of flocs, promoting floc growth and improving consolidation behaviors.

The main objective of this paper was to investigate flocculation, rheology and consolidation behaviors of kaolin slurries at pH 8 under varied PEI dosage and Mw conditions by analyzing dominant inter-aggregate forces and floc microstructures governing slurries. PEI dosages were varied between $0.4, \quad 0.04$ and 0.004 dry weight percentages or dwb\% (g.polymer/100g.kaolin powder). A high PEI dosage of $0.4 \mathrm{dwb} \%$ was chosen primarily to display possible PEI dosage effects on the sedimentation of kaolin slurries. PEI Mw were varied between low Mw of 1,800g/mol and high Mw of 70,000g/mol and 750,000g/mol. Under these varied PEI dosages and/or $\mathrm{Mw}$ conditions, the effects of shear within consolidated kaolin sediment beds were analyzed by varying the number of shear revolutions between 6 and 18. Rake speeds were kept low and constant at 1.5rpm so as not to cause permanent damage to floc aggregates. Yield stress, sedimentation and shear measurements expressed differences in rheology and consolidation behaviors between kaolin slurries. Zeta potential, particle (or floc) size, turbidity measurements and cryogenic scanning electron microscopy (cryoSEM) imaging of these slurries substantiated these differences and inferences made concerning dominant inter-aggregate forces and floc microstructures within slurries. These inferences explained floc aggregate properties and consolidation and dewatering behaviors of these slurries.

\section{Materials, their Characterization and Methods of Analysis}

\subsection{Materials and their Characterization}


As-received kaolin sourced from Unimin Australia Ltd was used in this study. The BET surface area of Unimin kaolin measured using a Micromeritics Gemini 2360 Surface Area Analyzer using $\mathrm{N}_{2}$ absorption is $19.9 \mathrm{~m}^{2} / \mathrm{g}$. The natural $\mathrm{pH}$ of this kaolin suspension in water is 8 where its corresponding zeta potential measured on a Colloidal Dynamics ZetaProbe Analyzer is $-36.4 \mathrm{mV}$. The particle size distribution of Unimin powder measured using a Malvern Mastersizer Microplus particle size analyzer is shown in Table 1.

Table 1

Particle size characterization (in $\mu \mathrm{m}$ ) of Unimin kaolin powder.

\begin{tabular}{|l|l|l|l|l|l|}
\hline Kaolin type & $\mathrm{D}(4,3)$ & $\mathrm{D}(3,2)$ & $\mathrm{D}(\mathrm{v}, 0.9)$ & $\mathrm{D}(\mathrm{v}, 0.5)$ & $\mathrm{D}(\mathrm{v}, 0.1)$ \\
\hline Unimin & 5.44 & 1.30 & 12.60 & 3.91 & 0.44 \\
\hline
\end{tabular}

Unimin kaolin carries high amounts of calcium, Ca(II) cations in comparison to most other kaolinites such as Riedel, Sigma, Fluka or Meckering [25]. These cations alter the rheological and flocculation behaviors of Unimin kaolinites from behaviors exhibited by the other kaolinites with low $\mathrm{Ca}(\mathrm{II})$ concentrations [25]. $\mathrm{Ca}$ (II) cations cause yield stress peaks of Unimin slurries to be at $\mathrm{pH} \sim 8$ to 9 instead of $\mathrm{pH} 2$ (which occurs with the other kaolinites), improving slurry consolidation levels in the high $\mathrm{pH}$ region. Avadiar et al. [25] attributed these rheological and consolidation variations to unlike charge attractions propagated by $\mathrm{Ca}$ (II) cations or its hydrolysis products that exist in the high $\mathrm{pH}$ region within Unimin slurries. In most instances, with low concentrations of $\mathrm{Ca}$ (II) cations, kaolin particles are highly negatively charged in the high $\mathrm{pH}$ region, where yield stress is usually low and consolidation is poor.

In this study, branched PEI polymers with $\mathrm{Mw}$ of 1,800, 70,000 and 750,000 $\mathrm{g} / \mathrm{mol}$ were used. PEI Mw 1,800 and Mw 70,000 were obtained from Polysciences, Inc and PEI Mw 750,000 was obtained from Sigma-Aldrich Australia. The schematic representation of branched PEI molecules is depicted in Figure 1. The Mw of a PEI monomer is $43 \mathrm{~g} / \mathrm{mol}$. The 
number of repeating units in PEI of Mw 1,800, Mw 70,000 and Mw 750,000 is therefore 42, 1628 and $\sim 17442$ respectively. Within each PEI molecule, the proportion of primary, secondary and tertiary amine groups is 1:2:1. These PEI polymers are miscible in water.

\subsection{Methods of Analysis}

All kaolin slurries were prepared by adding appropriate amounts of kaolin powder to distilled water (and 0.1wt\% PEI stock solutions for yield stress measurements). Slurries were then sonicated using a Branson digital Sonifier with a $2.5 \mathrm{~cm}$ probe for $40-60$ seconds.

\subsubsection{Sedimentation and shear measurements}

For sedimentation, appropriate amounts of $0.1 \mathrm{wt} \%$ PEI stock solutions were then added into kaolin slurries. Slurry containers were placed onto a ball-mill roller which rotated at 36rpm for 5 minutes, after which slurry contents were emptied into 250ml beakers. At regular time intervals, mud-lines representing suspension-solution interfaces were measured to calculate solids content or weight percentages (wt\%). The initial solids content of slurries were $8 \mathrm{wt} \%$. Slurry conductivities were at $0.20 \mathrm{mS} / \mathrm{cm}$ and $\mathrm{pH}$ of slurries was at 8 . This is the $\mathrm{pH}$ of many mineral tailings. Upon completion of sedimentation, where no difference was observed between mud-lines with respect to time, mud-lines were measured, supernatants were removed and consolidated sediment beds were sheared at 1.5rpm for 6 or 18 revolutions using a rake shown (as an insert) in Figure 8. Simultaneously, torque measurements (in \%) that represent yield stresses of sediment beds were recorded on a Brookfield DV-II+P vane viscometer with the rake serving as a vane. Mud-lines of slurries were measured immediately after shear.

Single point zeta potential measurements were carried out on consolidated sediment beds using a Colloidal Dynamics ZetaProbe Analyzer and particle (or floc aggregate) sizes within consolidated sediment beds were measured using a Malvern Mastersizer Microplus particle 
size analyzer. Turbidity of supernatants was measured using a portable turbidimeter, Hanna Instruments, HI98703, which functions according to Method 180.1 of The Environmental Protection Agency of the United States of America, USEPA.

\subsubsection{Yield stress measurements}

For yield stress measurements, the initial $\mathrm{pH}$ of slurries were adjusted to 12 using 1-5M $\mathrm{NaOH}$ solutions. Subsequently, the $\mathrm{pH}$ of these slurries was decreased to 2 in a stepwise manner with the addition of $0.5-1.5 \mathrm{M} \mathrm{HCl}$ solutions where, correspondingly, yield stress measurements were taken.

Kaolin slurries prepared for yield stress measurements had an initial solids content of $40 \mathrm{wt} \%$ with conductivity of all slurries at $4.0 \mathrm{mS} / \mathrm{cm}$ at $22^{\circ} \mathrm{C}$. Yield stress was measured with Brookfield LVDV-II+P and RVDV-II+P vane viscometers, each having a spring constant (or torque at $100 \%$ scale reading) of $0.0673 \mathrm{mNm}$ and $0.7187 \mathrm{mNm}$ respectively. LVDV-II+P and RVDV-II+P vane viscometers are usually used to measure low to moderate yield stresses of slurries. The three-vane spindles were used where their rotational speeds on both viscometers were kept low and constant at 0.6rpm.

\subsubsection{Cryogenic scanning electron microscopy (cryo-SEM) imaging}

Kaolin slurry samples were prepared using cryogenic techniques for SEM imaging. These samples were obtained from consolidated sediment beds. For each sample, $100 \mu \mathrm{L}$ of slurry was enclosed within two brass planchets, each $3.0 \mathrm{~mm}$ diameter and $0.3 \mathrm{~mm}$ depth, and plunged into liquid nitrogen at temperatures of $-196^{\circ} \mathrm{C}$. This employment of small volumes of sample and the rapid plunging procedure minimizes ice crystal formation within samples, limiting volume changes and alterations of structures. Subsequently, planchets were mounted onto stages and transferred in a nitrogen environment to a Leica EM MED020 preparation system fitted with a Leica EM VCT100 control system. Vacuum levels within the preparation 
chamber were maintained at or below 1.1-1.2 * 10 $0^{-6}$ mbar. The temperature of the stage (and sample) was increased from $-140^{\circ} \mathrm{C}$ to $-100^{\circ} \mathrm{C}$ over 2 minutes and the samples left to stabilize to this temperature over 15 minutes. Subsequently, samples were fractured by knocking off the top planchet exposing a fresh surface of sample. Surface water was then sublimated at $100^{\circ} \mathrm{C}$ for 10 minutes, removing frozen water from only the upper exposed surface of the sample to reveal surface relief and structure. The sublimation time was optimized empirically, so that sufficient water had been removed to expose aggregate structures whilst maintaining the integrity of aggregate and pore structures. This differs from freeze-drying, where sublimation results in a fully dried sample where possible structural changes occur upon the removal of water. The fractured, sublimated sample was then coated with $6 \mathrm{~nm}$ of platinum $(\mathrm{Pt})$ and transferred under vacuum at liquid nitrogen temperatures to the cryo-SEM. Samples were imaged using a Zeiss 55 field emission SEM fitted with a Leica EM VCT100 cryo and anticontamination system. The SEM was operated at $3-5 \mathrm{kV}$ with a stage temperature of $-156^{\circ} \mathrm{C}$. Images were collected using the secondary electron detector.

\section{Results and Discussion}

\subsection{Flocculation, Rheology and Consolidation Behaviors of Kaolin Slurries: Effect of PEI}

\section{Flocculants}

In general, high solids content of Unimin kaolin sediment was obtained with low PEI dosages added, where PEI Mw did not affect solids content as such (Figure 2). Biggs et al. [13] also observed high flocculation efficiencies with low polymer concentrations added onto colloidal anionic polystyrene latex particles. In this study, for example, the solids content of Unimin kaolin slurries with $0.4 \mathrm{dwb} \%, 0.04 \mathrm{dwb} \%$ and $0.004 \mathrm{dwb} \%$ PEI of Mw 1,800 at $\mathrm{pH} 8$ were 11.8wt\%, 16.3wt\% and 16.8wt\% respectively. However, with 0.004dwb\% PEI Mw 1,800, Mw 70,000 and Mw 750,000, the solids content obtained were $16.8 \mathrm{wt} \%, 16.8 \mathrm{wt} \%$ and 
17.7wt\% respectively. This difference in solids content obtained was especially obvious between Unimin kaolin slurries with 0.4dwb\% PEI and slurries with lower PEI dosages of $0.04 \mathrm{dwb} \%$ and $0.004 \mathrm{dwb} \%$ at $\mathrm{pH} 8$, irrespective of PEI Mw.

At $\mathrm{pH}$ 8, pre-flocculated Unimin kaolin aggregates that form from weak interactions between kaolin particles and that carry free and available kaolin particle sites for interactions are net negatively charged with a zeta potential of $-36.4 \mathrm{mV}$ and conductivity of $0.20 \mathrm{mS} / \mathrm{cm}$. In contrast, at $\mathrm{pH}$ 8, (branched) PEI polymers that exist as relatively coiled structures and that carry free and available PEI polymer sites for interactions are slightly positively charged (Figure 3) where charges and configurations of branched PEI molecules identified correspond to slurry conditions studied at low ionic strengths and conductivities of $0.20 \mathrm{mS} / \mathrm{cm}$. PEI polymers gradually carry lower positive charge densities to transform from extended to coiled or more bulky configurations from the reducing intra-repulsive forces between interacting PEI particles on each polymer molecule as pH increases [18, 26, 27] (Figure 3).

At $\mathrm{pH}$ 8, unlike charge attractions, hydrogen bonding and van der Waals forces of attraction occur between the free and available sites on pre-flocculated Unimin kaolin aggregates and PEI molecules. However, due to the low positive charge densities on PEI molecules and as a result, weak electrostatic attractive forces between PEI molecules and kaolin particles, PEI molecules would adsorb weakly onto kaolin particle sites and would not lie flat on these particle sites at $\mathrm{pH}$ 8. Regardless of PEI Mw, with 0.4dwb\%, 0.04dwb\% and 0.004dwb\% PEI added, the surface coverage of Unimin kaolin particles were estimated to be $~ 35.5$, 355.4 and $\sim 3553.6 \AA^{2} /$ repeating unit of PEI molecule respectively where the surface coverage were calculated based on Unimin's total BET surface area at $19.9 \mathrm{~m}^{2} / \mathrm{g}$. While only a fraction of kaolin surfaces were covered by PEI molecules with $0.04 \mathrm{dwb} \%$ or $0.004 \mathrm{dwb} \%$ PEI added, $\sim 35.5 \AA^{2} /$ repeating unit of PEI molecule could represent high monolayer surface coverage of Unimin kaolin particles with $0.4 \mathrm{dwb} \%$ PEI added. This could indicate the neutralization of 
greater amounts of negative charges on Unimin kaolin particles, via weak unlike charge attractions, with $0.4 \mathrm{dwb} \% \mathrm{PEI}$ than with $0.04 \mathrm{dwb} \%$ or $0.004 \mathrm{dwb} \%$ PEI. This neutralization was confirmed by the lower (negative) zeta potential magnitudes of Unimin kaolin particles with $0.4 \mathrm{dwb} \%$ PEI than with $0.04 \mathrm{dwb} \%$ or $0.004 \mathrm{dwb} \%$ PEI at $\mathrm{pH} 8$.

From a zeta potential of $-36.4 \mathrm{mV}$ on Unimin kaolin particles without PEI at $\mathrm{pH}$ 8, zeta potentials of kaolin particles with 0.4dwb\% PEI Mw 1,800, Mw 70,000 and Mw 750,000 were registered at $-18.5 \mathrm{mV},-10.6 \mathrm{mV}$ and $-9.22 \mathrm{mV}$ respectively at $\mathrm{pH}$ 8. Zeta potentials of kaolin particles with $0.04 \mathrm{dwb} \%$ and $0.004 \mathrm{dwb} \%$ PEI Mw 750,000 were, however, registered at $-32.0 \mathrm{mV}$ and $-35.7 \mathrm{mV}$ respectively at $\mathrm{pH}$ 8. This could indicate that the amount of PEI that has been weakly adsorbed is high at high PEI dosages. Such weak adsorptions of PEI molecules onto kaolin particle sites could also result in free PEI polymer segments protruding into the slurries for further interactions where the concentrations of such weak PEI adsorptions and, as a result, protrusions will be higher with high PEI concentrations of $0.4 \mathrm{dwb} \%$ added than with $0.04 \mathrm{dwb} \%$ or $0.004 \mathrm{dwb} \%$ PEI added. The presence of such free PEI polymer segments that have associated with one another was observed from Figure 4a with $0.4 \mathrm{dwb} \% \mathrm{PEI}$ in contrast to Figure $4 \mathrm{~b}$ with $0.04 \mathrm{dwb} \%$ PEI where negligible amounts of un-interacted and free PEI segments were observed.

The presence of such numerous free PEI polymer segments protruding from flocculated kaolin aggregates could lead to electrosteric stabilization between these aggregates with 0.4dwb\% PEI added. This stabilization could contribute towards the poor supernatant turbidity observed within these slurries. While flocs grow in size to settle within slurries, small flocculated kaolin aggregates experience the effects of stabilization to remain suspended within supernatants, affecting supernatant clarities [28]. Supernatants only attain clarity over a significant period of time due to the very slow settling rates of these small aggregates. Table 2 shows turbidity values of respective slurries after 3 days of sedimentation 
where with $0.4 \mathrm{dwb} \%$ PEI turbidity was consistently higher than with $0.04 \mathrm{dwb} \%$ or 0.004dwb\% PEI.

Table 2

Supernatant turbidity (in Nephelometric Turbidity Units, NTU) of Unimin kaolin slurries with varying polyethylenimine (PEI) dosages and molecular weights (Mw) added at $\mathrm{pH} 8$.

\begin{tabular}{|c|c|c|c|c|c|c|c|c|c|}
\hline & \multicolumn{3}{|l|}{0.4} & \multicolumn{3}{|l|}{0.04} & \multicolumn{3}{|l|}{0.004} \\
\hline $\begin{array}{l}\text { PEI Mw } \\
(\mathrm{g} / \mathrm{mol})\end{array}$ & 1800 & 70000 & 750000 & 1800 & 70000 & 750000 & 1800 & 70000 & 750000 \\
\hline $\begin{array}{l}\text { Turbidity } \\
\text { (NTU) }\end{array}$ & 7.37 & 118 & 117 & 3.54 & 1.98 & 2.63 & 4.50 & 2.32 & 3.39 \\
\hline
\end{tabular}

A high PEI concentration of $0.4 \mathrm{dwb} \%$ could also affect floc microstructures formed within slurries (Figure 5). With 0.4dwb\% PEI, flocs adopt an open configuration with large amounts of intra-aggregate waters trapped within (Figure 5a). With $0.04 \mathrm{dwb} \% \mathrm{PEI}$, however, flocs adopt more compact configurations with smaller amounts of intra-aggregate waters trapped within (Figure 5b). As a result, fewer floc aggregates exist within a fixed area to support network structures within slurries with 0.4dwb\% PEI than with 0.04dwb\% PEI (Figure 5). This reflects towards the higher network strengths within and/or between floc aggregates with 0.4dwb\% PEI added than with 0.04dwb\% PEI added.

Particle (or floc) size analyses confirmed the variations in sizes and structures of flocs formed between these slurries. The average equivalent volume mean diameters or D $(4,3)$ of flocs formed with $0.4 \mathrm{dwb} \%$ PEI added were larger between 5.2 to $7.6 \mu \mathrm{m}$ compared to between 3.9 to $5.0 \mu \mathrm{m}$ with $0.04 \mathrm{dwb} \%$ and $0.004 \mathrm{dwb} \%$ PEI added. This reflects towards the larger volumes of water trapped within the open structured porous floc aggregates formed within the former slurries which, as a result, settled at higher (initial) settling rates [1, 29-31]. At 10 minutes into sedimentation, settling rates of flocs formed with $0.4 \mathrm{dwb} \%$ PEI Mw 70,000 and 
Mw 750,000 were $7.0 \mathrm{~cm}^{3} / \mathrm{min}$ and $6.3 \mathrm{~cm}^{3} / \mathrm{min}$ respectively compared to 1.0 to $2.0 \mathrm{~cm}^{3} / \mathrm{min}$ with $0.04 \mathrm{dwb} \%$ and $0.004 \mathrm{dwb} \%$ PEI.

The torque (or yield stress) values of consolidated sediment beds with $0.4 \mathrm{dwb} \%$ PEI and of beds with $0.04 \mathrm{dwb} \%$ or $0.004 \mathrm{dwb} \%$ PEI were also different. With $0.4 \mathrm{dwb} \% \mathrm{PEI}$, torque was higher between 20 to $32 \%$ compared to 10 to $14 \%$ with $0.04 \mathrm{dwb} \%$ or $0.004 \mathrm{dwb} \%$ PEI. Ray and Hogg [32] and Taylor et al. [5] made similar observations of increasing polymer dosages causing floc sizes to increase and specific rates of floc breakages to decrease significantly.

The yield stress of kaolin slurries at a given $\mathrm{pH}$, as shown in Figure 6, was measured after the stirring of slurries upon acid additions. This yield stress is a measure of the strength of interparticle forces in a flocculated network structure of clay particles and can be used as a reflection of the inter-aggregate forces between clay particle structures within sediment beds. These results re-emphasized the higher yield stresses of kaolin slurries with $0.4 \mathrm{dwb} \%$ PEI than of slurries with $0.04 \mathrm{dwb} \%$ or $0.004 \mathrm{dwb} \%$ PEI, irrespective of PEI Mw. For example, yield stress peaks of Unimin slurries with 0.4dwb\% PEI Mw 1,800 and Mw 750,000 were at 384.9Pa and 438.7Pa located at $\mathrm{pH} 6$ and 7.5 respectively compared to 96.2 to $137.7 \mathrm{~Pa}$ located at $\mathrm{pH}$ between 8 to 9.5 for slurries with $0.04 \mathrm{dwb} \%$ and $0.004 \mathrm{dwb} \%$ PEI.

The higher yield stresses reflect the stronger inter-aggregate and/or inter-layer attractive forces within sediment microstructures and/or bed layers with $0.4 \mathrm{dwb} \%$ PEI added than with $0.04 \mathrm{dwb} \%$ or $0.004 \mathrm{dwb} \%$ PEI added. While electrostatic interactions, hydrogen bonding or van der Waals forces of attraction do not yield floc aggregate structures with such high cohesion forces and network strengths, polymer bridging interactions and polymer-polymer chain attractions (or particle bridging interactions) result in aggregates which require large shear forces prior to rupture [13] and which could result in these less compact and consolidated sediment beds at $0.4 \mathrm{dwb} \% \mathrm{PEI}$ addition. 
However, it is explained that low concentrations of polymer bridging interactions occur at high polymer dosages or particle surface coverage, such as at $0.4 \mathrm{dwb} \% \mathrm{PEI}$ addition, where minimal or no bare surfaces that carry free particle sites are left for these interactions to occur [28, 33-36]. It is also explained that low concentrations of polymer bridging interactions occur at very low polymer dosages where there are insufficient polymer chains to propagate adequate bridging links between particles [34]. Concentrations of polymer-polymer chain attractions (or particle bridging interactions) will also be affected similarly by the dosages of polymers added. In the case of flat plates, which could represent the platelet-like structures of kaolin particles, optimum (polymer or particle) bridging flocculation occurs with half of the kaolin surface covered with polymer molecules [37]. In this case, the high surface coverage estimate of $\sim 35.5 \AA^{2} /$ repeating unit of PEI molecule at $0.4 \mathrm{dwb} \%$ PEI addition may not be factually correct as only about 20 - 30\% of amine groups on PEI molecules are positively charged at $\mathrm{pH}$ 8, leading to small portions of the filamentous polymer network structures on PEI segments adsorbing weakly via electrostatic attractions onto kaolin particle sites and large portions of PEI segments protruding into the slurry. The kaolin particle surface coverage at $0.4 \mathrm{dwb} \%$ PEI dosage is, as a result, low at $\mathrm{pH} 8$ favoring polymer bridging interactions and/or polymer-polymer chain attractions (or particle bridging interactions) within these slurries.

The high kaolin particle surface coverage estimate could be accurate within kaolin slurries with $0.4 \mathrm{dwb} \% \mathrm{PEI}$ at $\mathrm{pH} 2$ where high concentrations of PEI molecules carry high positive charge densities (Figure 3) to undergo significant numbers of adsorptions onto kaolin particle sites and exist in flat configurations on these particles, inducing kaolin particle flocculation via electrostatic interactions as explained by Gregory [15], Horn [16] and Alince and Van de Ven [17]. This reduces concentrations of free particle sites and causes significantly shorter polymer protrusions into the slurry, especially inhibiting polymer bridging interactions and 
polymer-polymer chain attractions within slurries with $0.4 \mathrm{dwb} \%$ PEI of high Mw of 70,000 and 750,000 . Within slurries with $0.4 \mathrm{dwb} \%$ PEI of low Mw of 1,800, polymer molecules would adsorb whole onto kaolin particle sites to form a positive patch that undergoes electrostatic interactions with another free kaolin particle site, inducing particle flocculation via electrostatic patch-charge attractions. High degrees of patch-charge attractions are, however, unlikely at high particle surface coverage at $\mathrm{pH} 2$ and at high $\mathrm{pH}$ of 8 where for the latter, PEI molecules carry low positive charge densities and kaolin particles carry high negative charge densities to inhibit the formation of positive patches and the probabilities of patch-charge attractions [38].

The initial collision rates between interacting sites on pre-flocculated kaolin aggregates and PEI molecules are higher with high kaolin particle concentrations at 8wt\% existing together with high PEI polymer concentrations at $0.4 \mathrm{dwb} \%$ compared to with low PEI concentrations at $0.04 \mathrm{dwb} \%$ and $0.004 \mathrm{dwb} \%$. Referring to Figure 7 as adapted from Gregory [28, 39], polymers extend further from aggregate particle surfaces after collisions and upon adsorptions (after stage $b$ ) than at equilibrium (after stage $c$ ) where polymers relax and reconform to their equilibrium states depending on the adsorption affinity of polymer segments for particle surfaces [13]. It is suggested that the average time between collisions is shorter than the time required for polymer re-conformations leading towards non-equilibrium flocculation or stage $f$ after stage $b$ (Figure 7) within slurries with $0.4 \mathrm{dwb} \%$ PEI. Stage $f$ (Figure 7) involves a free and extended PEI polymer segment colliding and adsorbing onto 2 or more sites on pre-flocculated kaolin aggregates where collisions with extended polymer chains increase rates and extent of polymer bridging interactions and polymer-polymer chain attractions (or particle bridging interactions) during the primary stages of particle flocculation within kaolin slurries with $0.4 \mathrm{dwb} \% \mathrm{PEI}$ at $\mathrm{pH} 8$ [13]. 
Polymer bridging interactions occur via unlike charge attractions, van der Waals forces of attraction and/or hydrogen bonding between branched PEI molecules at $0.4 \mathrm{dwb} \%$ of high Mw of 70,000 or 750,000 and free, charge stabilized particle sites on pre-flocculated kaolin aggregates at pH 8 [40]. Polymer-polymer chain attractions or intermolecular interactions between uncharged or weakly (positively) charged PEI segments via hydrophobic interactions, van der Waals forces of attraction and/or hydrogen bonding are also pronounced within these slurries due to the high fractions and the large protrusions of unadsorbed (free) and/or uncharged polymer segments on PEI Mw 70,000 or 750,000 molecules. At low charge densities as such, PEI molecules are thermodynamically less stable, allowing associations between PEI segments via hydrophobic interactions just like the formation of dimers commonly found in many polar organic molecules. Interacting PEI segments could be unadsorbed (free) or adsorbed onto kaolin particle sites via hydrogen bonding and/or van der Waals forces of attraction where associations between adsorbed PEI polymer segments lead to particle bridging interactions. The formation of highly branched and filamentous polymer network structures with spider-web morphologies, within which intra-aggregate pore spaces exist, was observed within slurries with 0.4dwb\% PEI Mw 750,000 (Figure 4a) due to these polymer-polymer chain attractions between the unadsorbed (free) PEI segments.

The presence of polymer bridging interactions and polymer-polymer chain attractions, as dominant inter-aggregate forces, allow the growth of flocs and the entrapment of large intraaggregate pore spaces within slurries with $0.4 \mathrm{dwb} \%$ PEI Mw 70,000 or 750,000 . Due to the high affinity between water molecules and kaolin particles via hydrogen bonding and van der Waals forces of attraction, large amounts of water are entrapped within these pore spaces to result in the formation of large and dense flocs which settle quick as open structured aggregates with high intra-aggregate waters and thus low solids content (Figure 5a). Due to the large floc sizes, few flocs exist within a fixed slurry area. However, due to these dominant 
inter-aggregate forces creating complex particle-polymer network structures, these few large flocs support themselves to yield at high stresses. Taylor et al. [5] supported this, explaining that at high polymer dosages, more bridging links are created and, as a result, floc strengths are increased.

However, lower extents of polymer bridging interactions are inferred to occur within slurries with $0.4 \mathrm{dwb} \% \mathrm{PEI}$ of low $\mathrm{Mw}$ of 1,800 at $\mathrm{pH}$. This is due to the smaller polymer molecular size of PEI Mw 1,800 which protrudes over shorter slurry areas upon adsorptions onto kaolin particle surfaces (even though these polymers are extended at stage $f$ after stage $b$ (Figure 7)), reducing probabilities of collisions and bridging interactions with other free kaolin particle sites. Polymer-polymer chain attractions are, however, suggested to be the dominant interaggregate force within these slurries due to the high fractions of free polymer segments on PEI molecules at high PEI dosages of $0.4 \mathrm{dwb} \%$. The reduced extents of polymer bridging interactions and the shorter polymer molecular extensions but the high fractions of free PEI segments during polymer-polymer chain attractions result in the formation of small, numerous flocs which carry small but numerous intra-aggregate pore waters spaces to result in the low solids content of these slurries, similar to the solids content of slurries with 0.4dwb\% PEI 70,000 or 750,000. The low concentrations of polymer bridging interactions were not substantial enough to reduce the yield stresses of these slurries due to the high concentrations of polymer-polymer chain attractions that result in the complex filamentous polymer-polymer network structures and high yield stresses of these slurries, similar to the yield stresses of slurries with $0.4 \mathrm{dwb} \%$ PEI Mw 70,000 or 750,000.

Particle (or floc) size, settling rates and turbidity results explain the formation of smaller flocs with $0.4 \mathrm{dwb} \%$ PEI Mw 1,800 added than with $0.4 \mathrm{dwb} \%$ PEI Mw 70,000 or 750,000 added. The D $(4,3)$ of flocs formed with $0.4 \mathrm{dwb} \%$ PEI Mw 1,800 were smaller at 5.2 $\mu \mathrm{m}$ compared to $6.4 \mu \mathrm{m}$ and $7.6 \mu \mathrm{m}$ with $0.4 \mathrm{dwb} \%$ PEI Mw 70,000 and Mw 750,000 respectively. Smaller 
flocs settle over a longer period of time where flocs formed within slurries with $0.4 \mathrm{dwb} \%$ PEI Mw 1,800 settled slower at $1.7 \mathrm{~cm}^{3} / \mathrm{min}$ compared to $7.0 \mathrm{~cm}^{3} / \mathrm{min}$ and $6.3 \mathrm{~cm}^{3} / \mathrm{min}$ within slurries with 0.4dwb\% PEI Mw 70,000 and Mw 750,000 respectively. The lower floc settling rates within slurries with $0.4 \mathrm{dwb} \%$ PEI $\mathrm{Mw}$ 1,800 increase the duration for possible collisions and flocculation of small kaolin aggregates, which are suspended within supernatants due to the electrosteric stabilization within slurries with $0.4 \mathrm{dwb} \%$ PEI. This reduces the supernatant turbidity of these slurries to $7.37 \mathrm{NTU}$ compared to $118 \mathrm{NTU}$ and 117NTU within slurries with 0.4dwb\% PEI Mw 70,000 and Mw 750,000 respectively. The extent of electrosteric stabilization and thus supernatant turbidity could also be higher within the latter slurries due to the larger protrusions of free polymer segments on PEI Mw 70,000 or 750,000 molecules than on PEI Mw 1,800 molecules.

The slight variations in dominant inter-aggregate forces and flocculation mechanisms induced slight differences in consolidation behaviors, in terms of settling rates and supernatant turbidity between slurries with $0.4 \mathrm{dwb} \%$ PEI of high Mw of 70,000 or 750,000 and of low Mw of 1,800. However, these variations in dominant inter-aggregate forces did not result in differences in the yield stress (or rheology) and solids content (or consolidation) of these slurries and this is attributed to the minor microstructural and negligible macro-structural variations between floc structures formed within these slurries. The macro-structural differences could have evened out where the effect of PEI Mw is inconsequential on the rheological and consolidation behaviors of these slurries. As $\mathrm{Yu}$ and Somasundaran [41] explained, the bridging effect could be insignificant even with high Mw polymers added.

PEI dosages, however, affected the rheological and consolidation behaviors between kaolin slurries. At low PEI concentrations of $0.04 \mathrm{dwb} \%$ and $0.004 \mathrm{dwb} \%$, where the effect of PEI Mw was negligible, initial collision rates between sites on pre-flocculated kaolin aggregates and PEI molecules are lower than PEI polymer re-conformation rates upon adsorptions onto 
kaolin particle sites. This leads to equilibrium flocculation as represented by stages $a, b, c$ and $d$ (Figure 7) where slightly coiled and branched PEI polymer segments at pH 8 (Figure 3) undergo re-conformation at stage $c$ (Figure 7) to exist in flatter configurations. These reduced extensions of PEI segments into the slurry and the absence of free PEI polymer chains to propagate adequate bridging links between particles at these low PEI dosages significantly reduce the concentrations of polymer bridging interactions and polymer-polymer chain attractions to reduce the yield stress with $0.04 \mathrm{dwb} \%$ or $0.004 \mathrm{dwb} \%$ PEI added compared to with $0.4 \mathrm{dwb} \% \mathrm{PEI}$ added [34]. Absence of these interactions also reduce the growth of flocs where flocs are small and more compact, trapping low amounts of intra-aggregate waters within (Figure 5b). This increases the solids content of these slurries in relation to slurries with $0.4 \mathrm{dwb} \%$ PEI. Nasser and James [3] reported similar results of sediment beds with strong attractive forces yielding low solids content compared to sediment beds with weak inter-aggregate forces.

Despite the higher solids content of Unimin kaolin slurries with $0.04 \mathrm{dwb} \%$ and $0.004 \mathrm{dwb} \%$ PEI added than with $0.4 \mathrm{dwb} \% \mathrm{PEI}$ added, the solids content of the former were similar to that of Unimin kaolin slurries without PEI flocculants. For example, the solids content of Unimin kaolin slurries without PEI was $16.5 \mathrm{wt} \%$ similar to $16.5 \mathrm{wt} \%$ and $17.7 \mathrm{wt} \%$ with $0.04 \mathrm{dwb} \%$ and 0.004dwb\% PEI Mw 750,000 respectively added at $\mathrm{pH}$ 8. Zeta potential, settling rates, turbidity, D (4,3), torque and yield stress parameters of Unimin slurries without PEI were also similar at $-36.4 \mathrm{mV}, 1 \mathrm{~cm}^{3} / \mathrm{min}, 2 \mathrm{NTU}, 4.15 \mu \mathrm{m}, 10.5 \%$ and $135.3 \mathrm{~Pa}$ respectively to slurries with $0.04 \mathrm{dwb} \%$ and $0.004 \mathrm{dwb} \% \mathrm{PEI}$, irrespective of PEI Mw. This explains that the amounts of PEI adsorbed by kaolin particles at $0.04 \mathrm{dwb} \%$ and $0.004 \mathrm{dwb} \%$ PEI addition are not significant enough to cause major variations in dominant inter-aggregate forces that govern slurry behaviors. Similar to Unimin kaolin slurries without PEI, the dominant interaggregate forces within these slurries with low PEI dosages could remain to be hydrogen 
bonding and van der Waals forces of attraction and not electrostatic interactions, due to the low concentrations of positively charged interacting particles on PEI molecules at $\mathrm{pH}$ 8, or polymer-polymer chain attractions, despite adsorbed filamentous PEI polymers attaching onto free kaolin particle sites to induce particle bridging interactions (Figure 4b).

\subsection{Shear Effects and Dewatering Behaviors of Kaolin Slurries: Effect of PEI Flocculants}

Consolidated sediment bed responses to shear re-instated relationships established between PEI dosages analyzed and corresponding floc microstructures formed and their strengths. Depending on floc aggregate packing within consolidated beds, variations in shear durations via alterations to numbers of shear revolutions affected slurry dewatering levels as observed in Figure 8. Low numbers of 6 revolutions were sufficient to affect positively the dewatering abilities of sediment beds with $0.04 \mathrm{dwb} \%$ and $0.004 \mathrm{dwb} \%$ PEI. For example, from a consolidated sediment bed solids content of $15.9 \mathrm{wt} \%$, after 6 and 18 shear revolutions within sediment beds with $0.04 \mathrm{dwb} \%$ PEI Mw 750,000, solids content obtained were $18 \mathrm{wt} \%$ and $16.5 w t \%$ respectively. In contrast, high numbers of 18 shear revolutions were required to cause considerable redistributions of aggregated flocs within sediment beds with $0.4 \mathrm{dwb} \%$ PEI that was not achievable with 6 shear revolutions. For example, from a consolidated sediment bed solids content of $12.5 \mathrm{wt} \%$, with 6 and 18 shear revolutions within sediment beds with $0.4 \mathrm{dwb} \%$ PEI Mw 750,000, final solids content obtained were $12.7 \mathrm{wt} \%$ and 14.5wt\% respectively. This trend is consistent within sediment beds with PEI Mw 1,800 and Mw 70,000.

Shear rates experienced by flocs within sediment beds were close to zero from the low rake rotation speeds of $1.5 \mathrm{rpm}$ employed. This mechanical rake movement breaks the interconnected bonds created between flocs within loose network structures in sediment beds. These loose network structures were formed by floc aggregates falling and resting on one 
another. The rake movement also results in low pressure zone pore spaces within sediment beds that are filled by water that escapes from ruptured flocs [23]. With longer shear durations of 18 revolutions or more, mechanical energy breaks more and/or stronger floc interactions created during flocculation and compaction of sediment beds. This allows the expulsion of more intra-aggregate waters into low pressure zones created, resulting in more dewatering and greater re-arrangements of flocs. Thus the raking process over 18 revolutions was observed to be especially beneficial to the open structured, voluminous flocs within sediment beds with $0.4 \mathrm{dwb} \%$ PEI. However, floc structures formed within sediment beds with $0.04 \mathrm{dwb} \%$ and $0.004 \mathrm{dwb} \%$ PEI could begin to experience permanent damage upon 18 shear revolutions. This is due to the extensive breakage of interactions between these flocs that prevents them from adopting compact forms. Instead, these disintegrated kaolin floc aggregates could begin to trap more water which could result in reductions in the final solids content obtained.

\section{Conclusions}

Relationships between flocculation mechanisms, rheology and consolidation behaviors of kaolin slurries were explained under varied PEI dosage and Mw conditions. This study provides insight to the significant effect of high PEI dosages $(0.4 \mathrm{dwb} \%$ PEI with respect to 8wt\% kaolin) on the consolidation behaviors of Unimin kaolin slurries at $\mathrm{pH}$ 8. At high PEI dosages of $0.4 \mathrm{dwb} \%$, zeta potentials, turbidity, floc settling rates, floc (particle) sizes, yield stresses (or rheology) and solids content (or consolidation) were substantially altered from when low PEI dosages of $0.04 \mathrm{dwb} \%$ and $0.004 \mathrm{dwb} \%$ were added into kaolin slurries or when no PEI was added. Flocculation mechanisms, which were affected by these high PEI dosages within kaolin slurries, controlled dominant inter-aggregate forces and properties of floc microstructures formed between pre-flocculated kaolin aggregates and PEI molecules. In this case, non-equilibrium flocculation occurred, propagating polymer bridging interactions and 
polymer-polymer chain attractions within slurries with $0.4 \mathrm{dwb} \%$ PEI Mw 70,000 or 750,000 and polymer-polymer chain attractions within slurries with $0.4 \mathrm{dwb} \%$ PEI Mw 1,800 as dominant inter-aggregate forces, which resulted in strong flocs with high yield stresses and low solids content. However, interestingly, the effect of PEI Mw on the rheological and consolidation behaviors of kaolin slurries was observed to be negligible. The presence of polymer-polymer chain attractions was observed for the first time in cryogenic SEM images presented in this paper.

\section{Acknowledgements}

We wish to acknowledge The Australian Research Council (ARC) for funding this project via DP1096528 and Peta L. Clode and Lyn Kirilak from the Centre for Microscopy, Characterisation and Analysis (CMCA), University of Western Australia (UWA) for their guidance in preparing samples for cryogenic-SEM imaging. We wish to thank the reviewers for making this a better paper.

\section{References}

[1] R. Hogg, Flocculation and dewatering, Int. J. Miner. Process. 58 (2000) 223-236. [2] P. Mpofu, J. Addai-Mensah, J. Ralston, Investigation of the effect of polymer structure type on flocculation, rheology and dewatering behaviour of kaolinite dispersions, Int. J. Miner. Process. 71 (2003) 247-268.

[3] M.S. Nasser, A.E. James, The effect of polyacrylamide charge density and molecular weight on the flocculation and sedimentation behaviour of kaolinite suspensions, Sep. Purif. Technol. 52 (2006) 241-252.

[4] J. Addai-Mensah, Enhanced flocculation and dewatering of clay mineral dispersions, Powder Technol. 179 (2007) 73-78.

[5] M.L. Taylor, G.E. Morris, P.G. Self, R.S.C. Smart, Kinetics of Adsorption of High Molecular Weight Anionic Polyacrylamide onto Kaolinite: The Flocculation Process, J. Colloid Interface Sci. 250 (2002) 28-36.

[6] A.J. McFarlane, K.E. Bremmell, J. Addai-Mensah, Optimising the dewatering behaviour of clay tailings through interfacial chemistry, orthokinetic flocculation and controlled shear, Powder Technol. 160 (2005) 27-34.

[7] A. McFarlane, K.Y. Yeap, K. Bremmell, J. Addai-Mensah, The influence of flocculant adsorption kinetics on the dewaterability of kaolinite and smectite clay mineral dispersions, Colloids Surf. A 317 (2008) 39-48.

[8] L. Besra, D.K. Sengupta, S.K. Roy, P. Ay, Flocculation and dewatering of kaolin suspensions in the presence of polyacrylamide and surfactants, Int. J. Miner. Process. 66 (2002) 203-232. 
[9] L. Besra, D.K. Sengupta, S.K. Roy, P. Ay, Influence of polymer adsorption and conformation on flocculation and dewatering of kaolin suspension, Sep. Purif. Technol. 37 (2004) 231-246.

[10] L. Besra, D.K. Sengupta, S.K. Roy, Influence of unadsorbed and weakly adsorbed flocculants on separation properties of kaolin suspensions, Int. J. Miner. Process. 78 (2006) 101-109.

[11] J. Addai-Mensah, K.Y. Yeap, A.J. McFarlane, The influential role of pulp chemistry, flocculant structure type and shear rate on dewaterability of kaolinite and smectite clay dispersions under couette Taylor flow conditions, Powder Technol. 179 (2007) 79-83. [12] M.J. Pearse, Historical use and future development of chemicals for solid-liquid separation in the mineral processing industry, Miner. Eng. 16 (2003) 103-108.

[13] S. Biggs, M. Habgood, G.J. Jameson, Y.d. Yan, Aggregate structures formed via a bridging flocculation mechanism, Chem. Eng. J. 80 (2000) 13-22.

[14] J. Swenson, M.V. Smalley, H.L.M. Hatharasinghe, Structure of bridging polymers, J. Chem. Phys. 110 (1999) 9750-9756.

[15] J. Gregory, The effect of cationic polymers on the colloidal stability of latex particles, J. Colloid Interface Sci. 55 (1976) 35-44.

[16] D. Horn, Poly (ethylenimine)-physicochemical properties and applications, in: E.J. Goethals (Ed.), Polymeric amines and ammonium salts, Pergamon Press, Oxford, UK, 1980, pp. 333-355.

[17] B. Alince, T.G.M. Van De Ven, Stability of Clay Suspensions-Effect of pH and Polyethylenimine, J. Colloid Interface Sci. 155 (1993) 465-470.

[18] R.I.S. Gill, T.M. Herrington, The flocculation of kaolin suspensions using polyethylenimine and cationic polyacrylamides of the same molar mass but different charge density, Colloids Surf. 28 (1987) 41-52.

[19] A. McFarlane, K. Bremmell, J. Addai-Mensah, Microstructure, rheology and dewatering behaviour of smectite dispersions during orthokinetic flocculation, Miner. Eng. 18 (2005) 1173-1182.

[20] D.V. Boger, P.J. Scales, F. Sofra, Rheological concepts, in: R.J. Jewell, A.B. Fourie (Eds.), Paste and thickened tailings: a guide, Australian Centre for Geomechanics, Perth, Australia, 2006, pp. 25-37.

[21] S.B. Johnson, D.R. Dixon, P.J. Scales, The electrokinetic and shear yield stress properties of kaolinite in the presence of aluminium ions, Colloids Surf. A 146 (1999) 281291.

[22] A.S. Michaels, J.C. Bolger, Settling Rates and Sediment Volumes of Flocculated Kaolin Suspensions, Ind. Eng. Chem. Fundam. 1 (1962) 24-33.

[23] J. Du, R.A. Pushkarova, R.S.C. Smart, A cryo-SEM study of aggregate and floc structure changes during clay settling and raking processes, Int. J. Miner. Process. 93 (2009) 66-72.

[24] J.B. Farrow, R.R.M. Johnston, K. Simic, J.D. Swift, Consolidation and aggregate densification during gravity thickening, Chem. Eng. J. 80 (2000) 141-148.

[25] L. Avadiar, Y.K. Leong, A. Fourie, T. Nugraha, Rheological response to CA(II) concentration - the source of kaolin slurry rheological variation, Chemeca 2012: Quality of life through chemical engineering, Wellington, N.Z., 2012, pp. 1034-1043.

[26] R. Mészáros, I. Varga, T. Gilányi, Adsorption of Poly(ethyleneimine) on Silica

Surfaces: Effect of pH on the Reversibility of Adsorption, Langmuir 20 (2004) 5026-5029. [27] X. Zhu, T. Uchikoshi, T.S. Suzuki, Y. Sakka, Effect of Polyethylenimine on Hydrolysis and Dispersion Properties of Aqueous Si3N4 Suspensions, J. Am. Ceram. Soc. 90 (2007) 797-804. 
[28] J. Gregory, Particles in Water : Properties and Separation Methods, CRC Press, London, 2005.

[29] P.K. Weissenborn, L.J. Warren, J.G. Dunn, Selective flocculation of ultrafine iron ore 2. Mechanism of selective flocculation, Colloids Surf. A 99 (1995) 29-43.

[30] J.B. Farrow, L.J. Warren, The measurement of floc density-floc size distributions, in:

B.M. Moudgil, B.J. Scheiner (Eds.), Flocculation and Dewatering, Engineering Foundation, New York, 1989, pp. 153-166.

[31] J.B. Farrow, L.J. Warren, A new technique for characterizing flocculated suspensions, Dewatering Technology and Practice Conference, Brisbane, Australia, 1989, pp. 61-64.

[32] D.T. Ray, R. Hogg, Agglomerate breakage in polymer-flocculated suspensions, J.

Colloid Interface Sci. 116 (1987) 256-268.

[33] J. Swenson, M.V. Smalley, H.L.M. Hatharasinghe, Mechanism and Strength of Polymer Bridging Flocculation, Phys. Rev. Lett. 81 (1998) 5840-5843.

[34] T. Tripathy, B.R. De, Flocculation: a new way to treat the waste water, J. Phys. Sci. 10 (2006) 93-127.

[35] L.T. Lee, R. Rahbari, J. Lecourtier, G. Chauveteau, Adsorption of polyacrylamides on the different faces of kaolinites, J. Colloid Interface Sci. 147 (1991) 351-357.

[36] L. Nabzar, A. Carroy, E. Pefferkorn, Formation and properties of the kaolinitepolyacrylamide complex in aqueous media, Soil Sci. 141 (1986) 113-119.

[37] A.A. Zaman, Effects of Polymer Bridging and Electrostatics on the Rheological Behavior of Aqueous Colloidal Dispersions, Part. Part. Syst. Char. 20 (2003) 342-350.

[38] Y.K. Leong, Interparticle forces arising from an adsorbed strong polyelectrolyte in colloidal dispersions: charged patch attraction, Colloid. Polym. Sci. 277 (1999) 299-305. [39] J. Gregory, Polymer adsorption and flocculation in sheared suspensions, Colloids Surf. 31 (1988) 231-253.

[40] F. Csempesz, S. Rohrsetzer, The effect of polymer bridging on the flocculation kinetics of colloidal dispersions, Colloids Surf. 31 (1988) 215-230.

[41] X. Yu, P. Somasundaran, Role of Polymer Conformation in Interparticle-Bridging Dominated Flocculation, J. Colloid Interface Sci. 177 (1996) 283-287. 


\section{Figure captions}

Fig. 1. Schematic representation of branched polyethylenimine (PEI) molecules.

Fig. 2. Sedimentation behaviors: Solids content (wt\%) of kaolin slurries vs. time (min) with varying polyethylenimine (PEI) dosages and molecular weights $(\mathrm{Mw})$ added into slurries at $\mathrm{pH} 8$. The plot 'Unimin only' was included for comparison. Unimin only ( $\mathbf{\nabla}), 0.4 \mathrm{dwb} \%$ PEI Mw 1,800

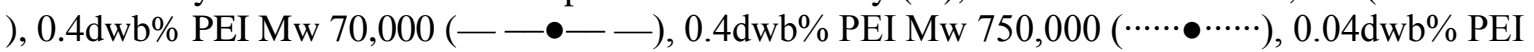
Mw 1,800 (- - $), 0.04 \mathrm{dwb} \%$ PEI Mw 70,000 (-- - - ), 0.04dwb\% PEI Mw 750,000 (ロ), $0.004 \mathrm{dwb} \%$ PEI Mw 1,800 ( $), 0.004 \mathrm{dwb} \%$ PEI Mw 70,000 ( $\mathbf{\Delta}), 0.004 \mathrm{dwb} \%$ PEI Mw 750,000 ($\cdot \cdot-\cdot \cdot \cdot)$.

Fig. 3. Schematic representation depicting variations in charges and configurations of branched polyethylenimine (PEI) molecules with respect to $\mathrm{pH}$.

Fig. 4. Extents of free and available polyethylenimine (PEI) polymer chains for further interactions within Unimin kaolin slurries at $\mathrm{pH} 8$ with (a) 0.4dwb\% PEI Mw 750,000 (at 15,000x) and (b) $0.04 \mathrm{dwb} \%$ PEI Mw 750,000 (at 16,000x). Scales are at $2 \mu \mathrm{m}$. The black areas depict frozen waters trapped within floc aggregates.

Fig. 5. Floc microstructures before shear within Unimin kaolin slurries at $\mathrm{pH} 8$ with (a) $0.4 \mathrm{dwb} \% \mathrm{PEI}$ Mw 750,000 and (b) 0.04dwb\% PEI Mw 750,000. Magnifications at 10,000x and scales at 5 $\mathrm{mm}$. The black areas depict frozen waters trapped within floc aggregates.

Fig. 6. Rheology behaviors: Yield stress $(\mathrm{Pa})-\mathrm{pH}$ trends of $40 \mathrm{wt} \%$ Unimin kaolin slurries with varying polyethylenimine (PEI) dosages and molecular weights (Mw) added. The plot 'Unimin only' was included for comparison. Unimin only ( $₫$ ), 0.004dwb\% PEI Mw 1,800 ( $)$, , 0.04dwb\% PEI Mw 1,800 ( $\mathbf{\Delta}), 0.4 \mathrm{dwb} \%$ PEI Mw 1,800 ( $\Delta$ ), 0.004dwb\% PEI Mw 750,000 (ロ), 0.04dwb\% PEI Mw 750,000 (口), 0.4dwb\% PEI Mw 750,000 (•).

Fig. 7. Schematic representation of sequential stages of polymer (branched molecules) adsorption onto pre-flocculated aggregates (platelet-like structures) and flocculation: (a) mixing between polymers and aggregates, (b) collision and adsorption of polymers onto faces and/or edges of aggregate particle surfaces, (c) polymer re-conformation to equilibrium state on aggregate particle surfaces, (d) aggregation of flocs or flocculation, (e) break-up of flocs and (f) non-equilibrium flocculation.

Fig. 8. Shear effects: Solids content (wt\%) of kaolin obtained before and after shear within slurries with PEI Mw 750,000 at pH 8 vs. PEI dosages (dwb\%) added into respective slurries. Before application of shear $(\bullet)$, after 6 shear revolutions $(\boldsymbol{\bullet})$, after 18 shear revolutions $(\boldsymbol{\Delta})$. Insert: Rake used to induce shear. 


\section{Vitae}

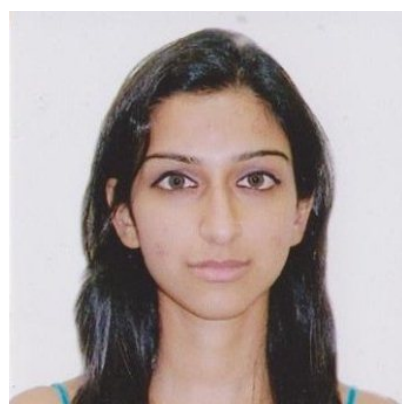

Lavanya Avadiar is a Bachelor of (Mechanical) Engineering (Honors) graduate from The University of Western Australia. She is currently part of a research team in the university as a postgraduate research student. Her interest lies in the study of rheology, surface chemistry, particle packing behaviors and microstructures of clay minerals under varied flocculant conditions, where one of her recent conference publications received The Graeme Jameson 2012 Award from the Chemeca 2012 conference.

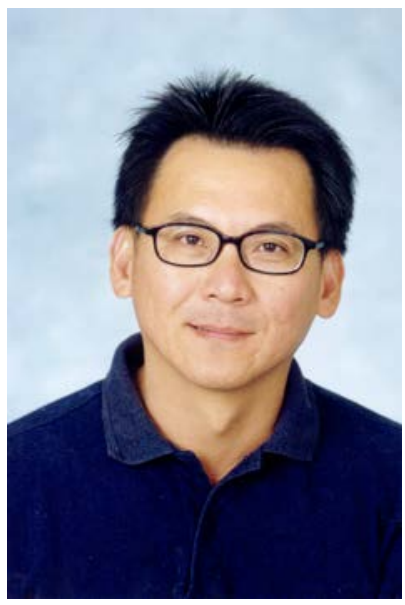

Yee-Kwong Leong obtained his PhD degree from the University of Melbourne. He is currently a professor in The University of Western Australia. His expertise involves the study of colloidal and nano-particulate processing via manipulations of surface forces and chemistry through the additions of flocculants to relate to rheology of dispersions and complex fluids. He has worked in this field for over 25 years and has more than 150 refereed articles. 


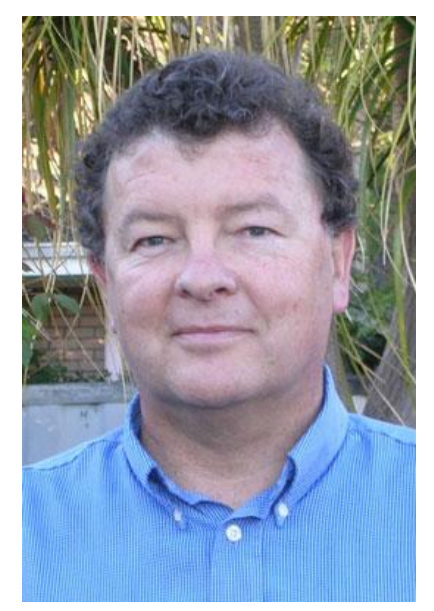

Andy Fourie, who is a PhD graduate, holds several positions such as the Head of School/Winthrop Professor in The University of Western Australia and as a Professional Fellow with the Australian Centre for Geomechanics. His expertise lies in the field of environmental geomechanics and mine tailings, where he has studied the dewatering and stabilization behaviors of soft clay mine tailings. He has worked in this field for 25 years where he has published over 100 refereed articles. 